\title{
Prediction of Ideas Number During a Brainstorming Session
}

\author{
Hassan Ait Haddou • Guy Camilleri • \\ Pascale Zaraté
}

\begin{abstract}
In this paper, we present an approach allowing the prediction of ideas number during a brainstorming session. This prediction is based on two dynamic models of brainstorming, the non-cognitive and the cognitive models proposed by Brown and Paulus (Small Group Res 27(1):91-114, 1996). These models describe for each participant, the evolution of ideas number over time, and are formalized by differential equations. Through solution functions of these models, we propose to calculate the number of ideas of each participant on any time intervals and thus in the future (called prediction). To be able to compute solution functions, it is necessary to determine the parameters of these models. In our approach, we use optimization model for model parameters calculation in which solution functions are approximated by numerical methods. We developed two generic optimization models, one based on Euler's and the other on the fourth order Runge-Kutta's numerical methods for the solving of differential equations, and we apply them to the non-cognitive and respectively to the cognitive models. Through some feasibility tests, we show the adequacy of the proposed approach to our prediction context.
\end{abstract}

Keywords Dynamic model · Brainstorming · Parameters calculation of dynamic models · Optimization models $\cdot$ Prediction

H. A. Haddou · G. Camilleri $(\varangle) \cdot$ P. Zaraté

Institut de Recherche en Informatique de Toulouse, SMAC, 118 Route de Narbonne, 31062,

Toulouse Cedex 9, France

e-mail: camiller@irit.fr

H. A. Haddou

e-mail: haithaddou@yahoo.com

P. Zaraté

e-mail: zarate@irit.fr 


\section{Introduction}

The brainstorming technique is a very popular group technique for generating ideas. It is not surprising that this technique is often used in group decision making meetings (GDMM). Our aim is to integrate some specifi support tools dedicated to facilitation activity into Group Decision Support Systems (GDSS) also called Group Support Systems (GSS). In our work we use the following definition of GDSS proposed by DeSancis et al.: "A GDSS is a computer-based technology designed to help committees, project teams, and other small groups with activities such as problem identificatio and analysis, decision making, planning, creativity, conflic management, negotiation, and meeting management. GDSSs combine communication, information, and decision support technologies in an integrated environment." p. 552 in DeSanctis et al. (2008). Many studies showed the advantage of using such systems in meetings in order to improve their effectiveness and efficien y (Nunamaker et al. 1991; de Vreede et al. 2003; Soller et al. 2005). Group facilitation is define as a process in which a person who is acceptable to all members of the group intervenes to help improving the way it identifie and solves problems, and makes decision (Schwarz 1994). Facilitation, on the other hand, is a dynamic process that involves managing relationships among people, tasks, and technology, as well as structuring tasks and contributing to the effective accomplishment of the meeting's outcomes (den Hengst and Adkins 2007). Facilitation was for a long time recognized as a key success factor for meetings (Bostrom et al. 1993; Schwarz 1994; Hayne 1999). When GDSS tools are used or when e-meetings take place, the need to facilitate meetings is even more visible (Viller 1991; Bostrom et al. 1993; Macaulay and Alabdulkarim 2005).

Unfortunately, due to their specifi skills, professional facilitators are mobile, scarce and expensive. Organizations have thus difficult to maintain a stable in-house facilitation capability (den Hengst and Adkins 2007; Briggs et al. 2010). Therefore, it seems relevant to support this activity in order to make it more accessible to facilitators with less expertise while maintaining performance comparable to those obtained by a skilled facilitator and thus promote the adoption of GDSS in organizations.

Many works integrated elements of automated facilitation in GDSS tools (Limayem and DeSanctis 2000; Nunamaker et al. 2002; Wong and Aiken 2003; Macaulay and Alabdulkarim 2005; Adla et al. 2011). These works show the interest of automating some facilitation elements. Studies such as Wong and Aiken (2003), Limayem (2006) further demonstrate that the integration of automated facilitation in GDSS can be as effective as using the same tools with skilled-human facilitation. It also enhances a faithfulness appropriation of the technology.

Our purpose is to offer assistance to the facilitator in real time during meetings. For this, it is necessary to analyze the group activity, or even anticipate (predict) its evolution for supporting the facilitator in her/his decision to intervene (when) and her/his choice of interventions type (what). In this work, we focus on a particular group technique, the brainstorming. We limit here our work to provide, in an automatic way, a justifie picture of the evolution of generated ideas for the current brainstorming activity in order to support the facilitator in her/his decision or choice of interventions. This information can be seen as a set of indications (or cues) on the dynamic of ideas generation (as in the work of Vivacqua et al. 2011). 
In this paper, we developed an approach that gives some information (predictions) about the evolution of ideas number over time that would be generated by participants at a brainstorming session. Our prediction approach is based on the dynamic models of brainstorming, that were presented by Brown and Paulus (1996). These models are formalized by differential equations describing the evolution of ideas number over time for each participant.

The group technique of brainstorming has been proposed by Osborn (1957). The purpose of this technique is to improve productivity and creativity of the group (i.e. bringing many different and original ideas). It is based on the four following rules:

- Criticism is ruled out. To avoid trouble in the generation of ideas, participants at a brainstorming session should not criticize. This rule follows a central principle of brainstorming: the "deferment of judgment".

- Freewheeling is welcomed. As it is not allowed to criticize, unusual and even wild ideas are possible and desired.

- Quantity is wanted. One purpose of brainstorming is to gather up a maximum number of ideas, assuming the greater number of ideas, higher is the probability to fin successful ones.

- Combination and improvement are sought. Participants are encouraged to use the ideas of others by combining or improving them.

In order to take the full advantage of brainstorming, Osborn mentioned that the previous rules were not sufficient In addition, he provided some suggestions and recommendations on the training and the management of brainstorming meetings (facilitation, participants training, topic nature, group composition, etc).

Osborn claimed that a real group using brainstorming technique to generate ideas will be more effective than individuals alone. He indicated that in a study, this technique generated $44 \%$ more useful ideas than individuals working alone. However, a large number of experimental studies contradict this claim by noting that interactive brainstorming groups tended to produce less than nominal groups (groups of similar size working alone) (Diehl and Stroebe 1987; Mullen et al. 1991).

Many researches have attempted to fin the reasons of the "failure" of interactive brainstorming (called productivity loss). They identifie four main reasons:

- Evaluation apprehension (Camacho and Paulus 1995; Mullen et al. 1991). Participants may feel a fear of negative evaluations from others. In this case, they may be led to not express their more original ideas (self-censorship).

- Social loafing or Free riding (Borgatta and Bales 1953; Diehl and Stroebe 1987; Karau and Williams 1993; Paulus and Dzindolet 1993). Social loafing is a loss of motivation and effort caused by the presence of others. Free riding occurs when an individual considers that his contribution is not necessary to the group success.

- Production blocking (Diehl and Stroebe 1991; Nijstad et al. 2003). When someone speaks, other participants are usually blocked, bringing about a risk of ideas forgetfulness.

- Matching. Participants in brainstorming session tend to want to produce as others (in the same order of magnitude). This behaviour seems to result from a comparison in terms of performance between individuals within a group. Brown and Paulus (1996) noted that matching may occur in different contexts in both directions, 
towards participants who are most productive when there is a climate of competition between group members (Upward matching Paulus and Dzindolet 1993), or conversely towards participants producing the least if motivation of the group is quite weak (Downward matching Borgatta and Bales 1953; Camacho and Paulus 1995; Karau and Williams 1993).

In order to improve the effectiveness of brainstorming meetings, many studies have attempted to reduce the loss of productivity. Camacho and Paulus (1995) formed groups with low anxious members; Oxley et al. (1996) showed the importance of trained facilitators which provide a control over free-riding; the use of electronic brainstorming for eliminating the blocking (Diehl and Stroebe 1991; Nijstad et al. 2003); anonymous electronic brainstorming in order to reduce evaluation apprehension (DeRosa et al. 2007); the addition of breaks in meetings and promoting divergent thinking (Brown and Paulus 2002; Coskun 2005), etc. Many finding of these studies seem to agree with the suggestions and the recommendations presented by Osborn (cf. the critical review of Isaksen (1998) emphasizing the importance of these suggestions and recommendations in brainstorming studies).

From works on productivity loss, Brown and Paulus (1996) proposed a modeling of ideas generation process in brainstorming. This modeling describes for each participant the evolution of ideas number over time according to the productions of other participants. It explicitly incorporates three social factors: the blocking, the matching (described above) and the decay. The decay factor is useful to decrease ideas number over time (Nijstad et al. 2003). In this modeling, the factors of evaluation apprehension and free riding are represented implicitly through values of several parameters. In this work, Brown and Paulus presented two models, a non-cognitive and a cognitive model. Of course, these models are a simplificatio of the ideas generation process, but are in line with finding of various studies on productivity loss. One interest of these models is to provide a clear and relatively precise description of the interdependence existing between participants' productions. In addition, they allow differentiating the impact of three social factors in ideas production. The initial objective of this modeling is to provide a framework for the development and the assessment of theoretical assumptions.

We believe that the model developed by Brown and Paulus can be adapted to our context of prediction. Indeed, assuming that the proposed dynamic models are completely determined (that is all parameters are valued), they offer for each participant an accurate description of ideas number represented in the form of mathematical function. By knowing this function of ideas number over time on its definition domain $D=[0,+\infty[$, it is possible for each participant to calculate on any subintervals of $D$ the ideas number and therefore, to compute an extrapolation of ideas number in the future (which we call prediction).

Coskun and Yilmaz (2009) improved the models proposed by Brown and Paulus by adding physical constraints (such as the rate of ideas is always positive) to make these models more realistic and complete. In addition, they modifie them to deal with two situations: the sequential situation where the problem is decomposed into sub-problems and each sub-problem is presented one after other to the group (called sequential brainstorming), and the situation of brainwriting. They also studied the impact of 
the instruction of ideas memorization on brainstormers. The aim of their work is to develop new models adapted to the previous situations (formalized by impulsive differential equations) and to show through experiments that these two models well fi to observations. In order to do so, they chose some values for model parameters so that models are close to their experiments.

Our approach towards dynamic models use is quite different from the above two studies. We determine ideas number from measurements of production duration of participants' ideas ${ }^{1}$ in real-time (that is ongoing the brainstorming session). For this, we must be able to compute relatively quickly the model parameters from ideas already expressed by participants. This context has motivated us to develop our approach for determining parameters of dynamic models presented in this work.

In order to make this approach directly usable (computable), we assume that each contribution of a participant matches to one and only one idea. This assumption may appear very strong implying the addition of the following rule "one idea per intervention", and the need to strictly ensure compliance with brainstorming rules. However, from preliminary experiments, it seems that the proposed approach remains useful for free ideation sessions. Nevertheless, more investigations must be achieved on this point.

First, we will present an overview of the proposed approach. In the second part, we will deal with the non-cognitive model. Our approach of parameters calculation is based on an optimization model. We will present a generic optimization model ${ }^{2}$ which will be applied to the non-cognitive model. Feasibility tests will show the adequacy of the proposed approach to our prediction context.

In the third part, the cognitive model will be presented. The optimization model developed for the non-cognitive can not be directly applied to the cognitive model. To cope with this problem, we will propose a more accurate (but more complex) new generic optimization model. This new problem will be adapted to the cognitive model, and as for the non-cognitve model, its feasibility will be tested. We will show that this new optimization model is well adapted to groups of small size, but may requires further developments for larger groups.

\section{Approach Overview}

The overall proposed process in this paper is schematically summarized in the Fig. 1. The box items represent problems and the oval-boxes the used approaches. The goal of this process is to determine time evolution functions of ideas production rate for each participant (box 1) from dynamic models (Ordinary Differential Equations ODE). In order to achieve this objective, two numerical methods (Euler and Runge-Kutta fourth RK4) are used (2). However, to be able to apply these methods, models parameters have to be known (3). A least-square optimization is used for computing these model parameters (4). From now, dynamic models and their parameters are known and

\footnotetext{
1 We intend to measure the duration of ideas production by using microphones.

2 We believe that this generic problem could be useful in many other situations.
} 


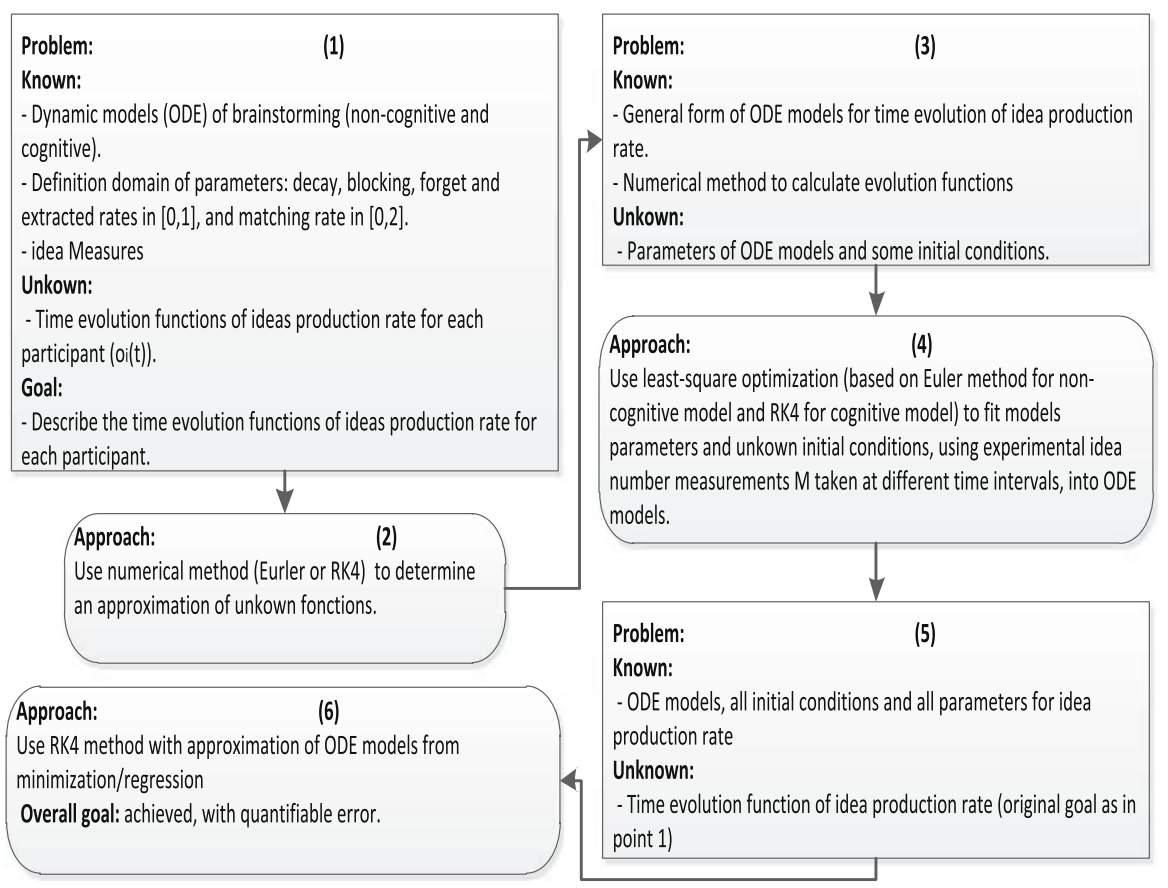

Fig. 1 Process overview

solution functions can be computed (5). Solution functions and a quantifiabl error are then determined by using the Runge-Kutta fourth (6).

\section{Non-Cognitive Model of Brainstorming}

In this part, the non-cognitive model is presented. We will begin with a description of the mathematical model (modifie by Coskun and Yimaz). Then a generic approach, using an optimization model to determine the parameters of dynamic models from measurements, will be presented. We will apply this generic optimization model to the non-cognitive model of brainstorming. In order to test the feasibility of our approach, we will use artificia tests. This part will end with a discussion on tests, advantages and disadvantages of the proposed approach.

\subsection{Mathematical Model}

The non-cognitive model is the simplest model presented by Brown and Paulus (1996). This model is based on the assumption that a participant's rate of idea production depends on the following three factors (see Brown and Paulus 1996):

1. Output decay: Any given participant will eventually run out of ideas. This factor serves to decrease productivity over time. 
2. Blocking: A participant's productivity will decrease as a function of total group output.

3. Matching: Participants adjust their productivity rate to more closely match the average group rate. This factor decreases a participant's productivity if it is higher than the group average and increases a participant's productivity if it is lower than the group average.

This model expresses for each participant, the variation of ideas rate as a weighted sum of decay, blocking and matching factors.

For a group of $n$ individuals $(i \in \llbracket 1, n \rrbracket)$, the non-cognitive model is formalized by the following system of differential equations:

$$
\begin{aligned}
& \frac{d o_{i}(t)}{d t}=-a_{i} o_{i}(t)-b_{i} \sum_{j \neq i} o_{j}(t)+m_{i}\left[\frac{1}{n-1} \sum_{j \neq i} o_{j}(t)-o_{i}(t)\right] \text { with } o_{i}(t) \geq 0 \\
& \text { and } t \in[0,+\infty[
\end{aligned}
$$

where $o_{i}(t)$ is the ideas rate (ideas number per time unit) generated by the participant $i, a_{i}$ the decay rate, $b_{i}$ the sensitivity to blocking, $m_{i}$ the tendency to match. Note that the rates $a_{i}, b_{i}$ and $m_{i}$ represent the impacts of decay, blocking and matching factors on the ideas rate of a participant $i$. This impact is assumed to be constant over time during the whole session (that is $a_{i}, b_{i}$ and $m_{i}$ are constant). The blocking is represented by bi times the sum of others' productions. The matching is estimated by the distance between productions of an individual and the average of others' productions. Following Coskun and Yilmaz (2009), we add the constraint $o_{i}(t) \geq 0$ to model the fact that ideas rate is always positive or null. A possible effect of this constraint is to break the linearity of the differential equations.

This model states that the ideas rate decreases at the rate $a_{i}$ with respect to its value, at the rate $b_{i}$ with respect to the sum of other's rates, and depending on cases decreases (negative value) at the rate $m_{i}$ if its value is higher than the average of others' rates (Downward matching) or increases (positive value) at the rate $m_{i}$ if its value is lower than the average (Upward matching).

For example, for a group of three persons, the cognitive model is:

$$
\left\{\begin{array}{l}
\frac{d o_{1}(t)}{d t}=-a_{1} o_{1}(t)-b_{1}\left(o_{2}(t)+o_{3}(t)\right)+m_{1}\left[\frac{1}{2}\left(o_{2}(t)+o_{3}(t)\right)-o_{1}(t)\right] \\
\text { with } o_{1}(t) \geq 0 \\
\frac{d o_{2}(t)}{d t}=-a_{2} o_{2}(t)-b_{2}\left(o_{1}(t)+o_{3}(t)\right)+m_{2}\left[\frac{1}{2}\left(o_{1}(t)+o_{3}(t)\right)-o_{2}(t)\right] \\
\text { with } o_{2}(t) \geq 0 \\
\frac{d o_{3}(t)}{d t}=-a_{3} o_{3}(t)-b_{3}\left(o_{1}(t)+o_{2}(t)\right)+m_{3}\left[\frac{1}{2}\left(o_{1}(t)+o_{2}(t)\right)-o_{3}(t)\right] \\
\text { with } o_{3}(t) \geq 0
\end{array}\right.
$$

The Fig. 2 illustrates the cognitive model for a group of three persons by drawing the ideas rate over time generated by each participant. In this example, the following parameters vectors have been used: $p_{1}=(3.337,0.044,0.140,0.222)$, $p_{2}=(3.467,0.005,0.022,0.256)$ and $p_{3}=(4.036,0.120,0.077,0.189)$ where 


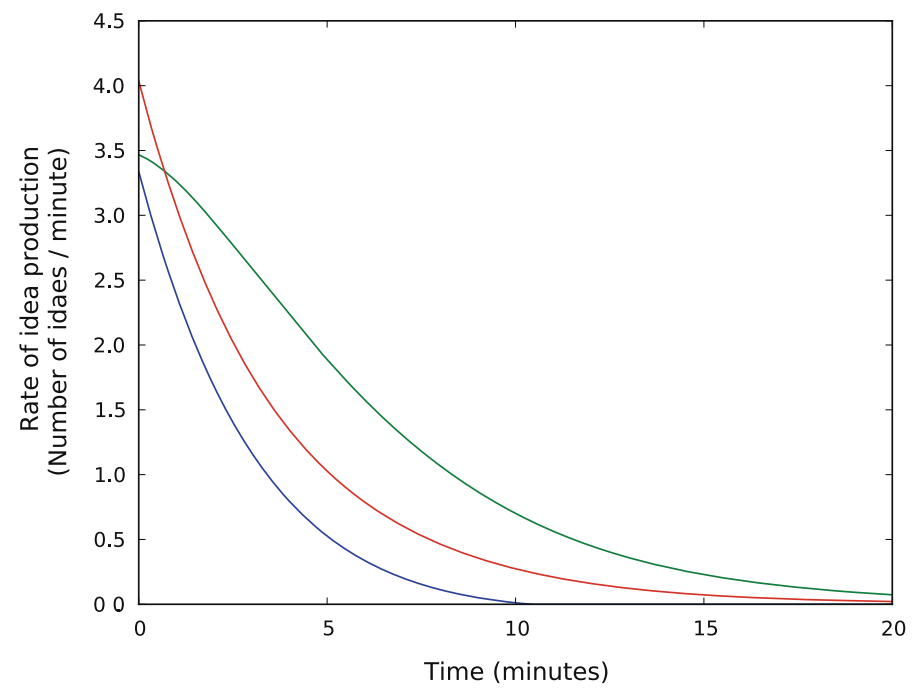

Fig. 2 Cognitive model for a group of three members (one curve by member)

$p_{i}=\left(o_{i}(0), a_{i}, b_{1}, m_{i}\right)$ is the parameters vector for participant $i$ with $o_{i}(0)$ the initial rate of ideas, $a_{i}, b_{i}$ and $m_{i}$ are respectively decay, blocking and matching rates.

The solutions of this system have been calculated by using the numerical method of Runge-Kutta order four ${ }^{3}$. In most cases, it is not possible to have an analytical solution due to equations complexity. Therefore, numerical methods can be used to calculate an approximation of solution functions.

\subsection{Euler Based Generic Optimization Model for Parameters Calculation}

This model is useful in a prediction context, which is used to calculate automatically model parameters only from measurements of production duration during the session. A natural way for parameters computing would be to start from analytical solution functions and through them to generate equations corresponding to measurements. Unfortunately, in every case analytical solutions are not necessarily known. In our approach, we propose to use optimization formulation for the calculation of the model parameters calculation in which solution functions are approximated by numerical methods.

For the non-cognitive model, we will use the Euler's method (Ait-Haddou et al. 2010). The Euler's method is a first-orde numerical procedure for solving ordinary differential equations with a given initial value. This method determines a linear approximation of the solution functions of firs order differential equations.

Let us consider the following firs order differential equation $\frac{d y}{d x}=f(x, y)$ with initial value $y(a)=y_{o}$ where $\mathrm{f}(\mathrm{x}, \mathrm{y})$ is a known function define on an interval $\mathrm{I}=[\mathrm{a}, \mathrm{b}]$.

\footnotetext{
3 With a step of $1 \mathrm{E}-3$.
} 
Let $h=\frac{b-a}{n}$ be a real positive number where $n$ is the number of considered points. The real $h$ is called "step" and it is the step sizes between the points $x_{0}, x_{1}, \ldots, x_{n}$.

The Euler's method considers two sequences of real numbers $x_{i}$ and $y_{i}$ for all $i \in \llbracket 0, n-1 \rrbracket$ such that $x_{i+1}=x_{i}+h$ and $y_{i+1}=y_{i}+h f\left(x_{i}, y_{i}\right)$ with values in initial condition are respectively $x_{0}=a$ and $y_{0}=y(a)$. The linear approximation function of $y(x)$ on $I$ is given by $\phi(x)=f\left(x_{i}, y_{i}\right) x+y_{i}-f\left(x_{i}, y_{i}\right) x_{i}$ for all $x \in\left[x_{i}, x_{i+1}\right]$ and $\phi\left(x_{0}\right)=y_{0}$.

In this work, we plan to determine the parameters of a system of differential equations obtained from measurements.

These measurements represent the number of ideas on a given interval $[\mathrm{a}, \mathrm{b}]$ which is equal to $\int_{a}^{b} y(t) d t$. To fin the parameters of dynamic models we propose the following optimization model:

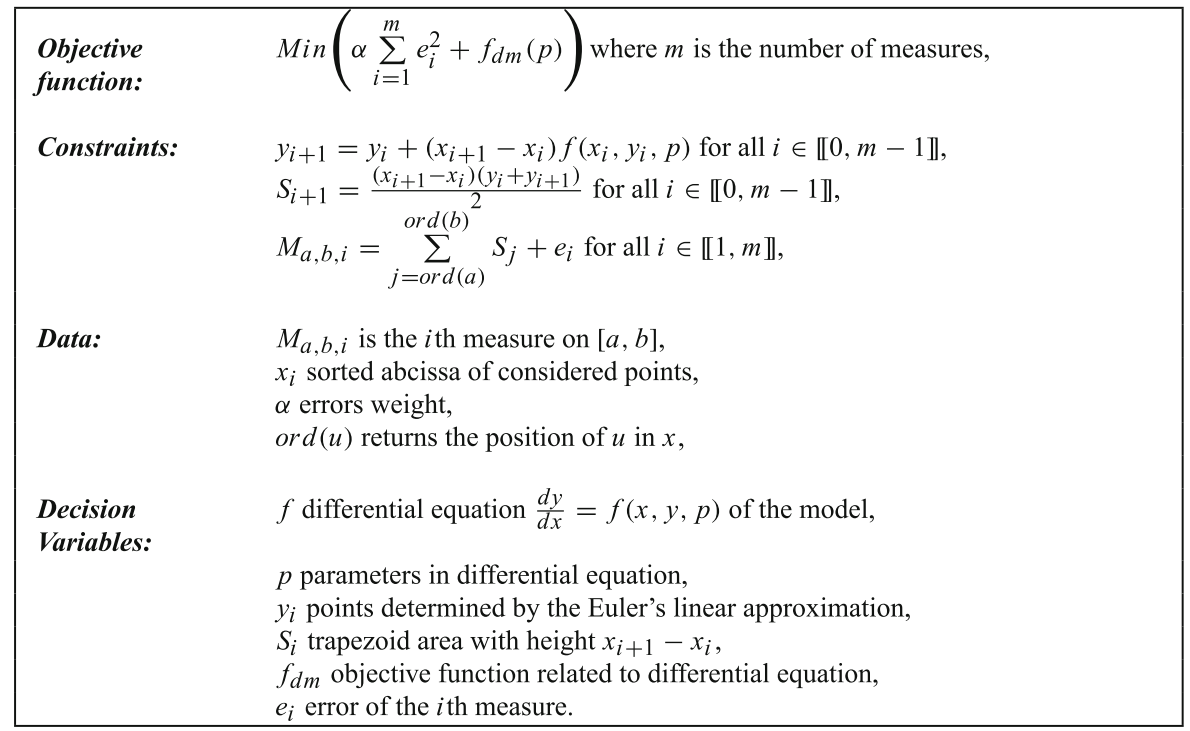

In this optimization model, the objective function is divided in two parts. As Euler's method computes an approximation of solution functions, an error is inevitably present. The firs part of objective function concerns the minimization of this error. The second part is specifi to the dynamic model considered. In constraints, $y_{i}$ are Euler's points, $S_{i}$ correspond to surfaces calculated with Euler's points. As Euler's method computes a linear approximation of solution functions; integration of these approximations corresponds to the calculation of trapezoid surfaces. In this problem, measures can overlap, therefore for one measure on $[a, b]$, it is possible that several points would be calculated, in this case the measure corresponding to $\int_{a}^{b} y(t) d t$ is equal to the sum of trapezoid surfaces define from these points. At each measure, an error is integrated for the approximation error but can also be used to introduce measurement errors. 


\subsection{Optimization Model for Parameters Calculation of Non-Cognitive Model}

In order to calculate parameters of the cognitive model, we apply the generic optimization model previously presented. In our prediction context, solving time is important to be able to use the optimization model during brainstorming sessions. Therefore, it is necessary to fin a good compromise between solving time and precision. For this, we chose as Euler's points the starting and the ending points of measure's intervals. In this way, we limit the number of decision variables such that $x_{i}, y_{i}$ and $S_{i}$ to the minimum entailing a simplificatio of the optimization model.

The optimization model for the non-cognitive model can be expressed as follow:

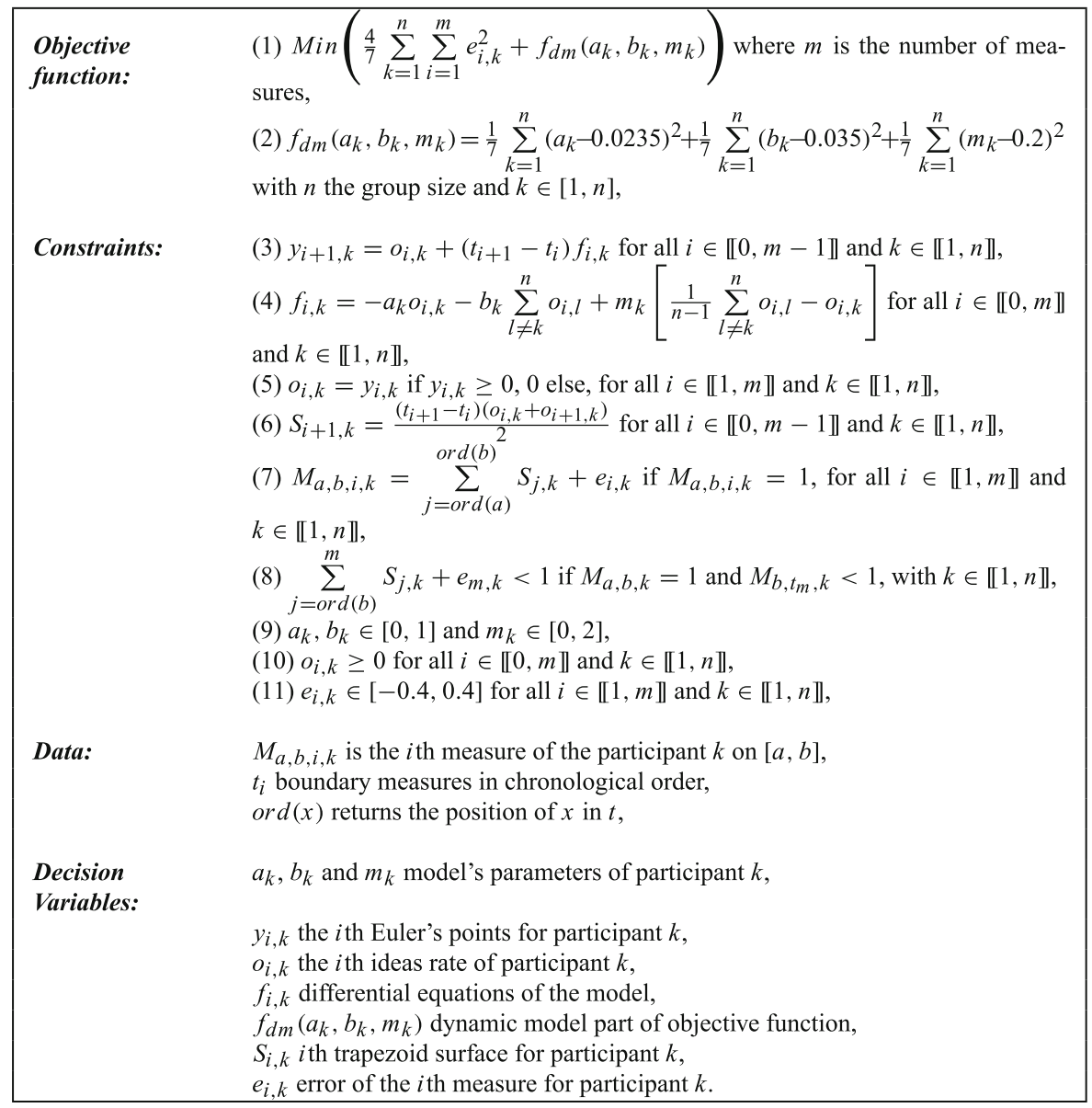

The model part of objective function (2) represents a square distance between social factors and their reference values. Reference values chose in this problem are those that have been established by Coskun and Yilmaz (2009) in their experimentations. The whole objective function is a weighted sum of square distances to reference values. 


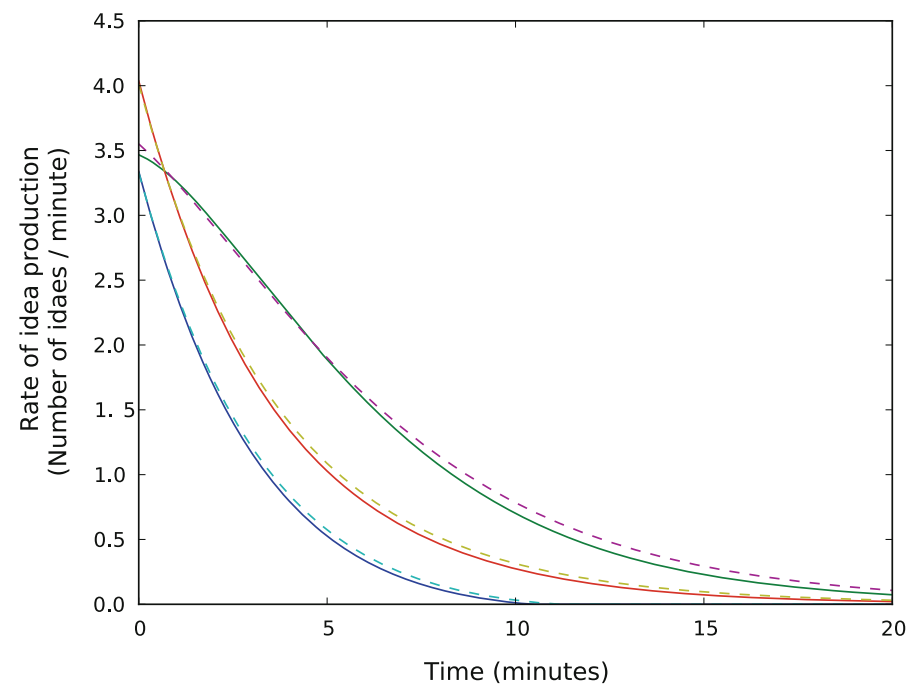

Fig. 3 Non-cognitive model with initial and found by optimization parameters

We consider that minimizing as possible errors is more important than factors' values close to their reference values, because more errors are low more measures follow the differential equations (model). This preference is translated in weights where errors have a weight of $\frac{4}{7}$ and social factors a weight of $\frac{1}{7}$.

The constraint (3) define Euler's points according to differential equations presents in (4). Ideas rates follow Euler's points if they are positive or are set to zero otherwise (5), this constraint comes from the Coskun's and Yilmaz's modelling. We chose to process every idea one by one, therefore our measures are define to one idea (7). When a brainstorming session finishes all participants could not have enough time to generate one more idea, this situation is formalized in the constraint (8).

For example in Fig. 3, full lines represent the initial ideas rates over time (in minutes) for a group of three participants. The initial model is graphed using Runge-Kutta's four order method with the same parameters as before (that is $p_{1}=$ $(3.337,0.044,0.140,0.222), p_{2}=(3.467,0.005,0.022,0.256)$ and $p_{3}=(4.036$, $0.120,0.077,0.189)$ ). From these initial solutions, measures are computed by determining for each participant, all consecutive intervals $[a, b] \subseteq[0,20]$ from 0 such as $\int_{a}^{b} o_{i}(t) d t=1$. We then use the non-cognitive optimization model only with these measures to calculate new parameters. The new parameters calculated in this way are $\hat{p}_{1}=(3.329,0.114,0.100,0.148), \hat{p}_{2}=(3.551,0.024,0.026,0.200)$ and $\hat{p}_{3}=(4.013,0.121,0.071,0.200)$ where $\hat{p}_{i}=\left(\hat{o}_{i}(0), \hat{a}_{i}, \hat{b}_{i}, \hat{m}_{i}\right)$ with $\hat{o}_{i}(0)$ the initial rate of ideas, $\hat{a}_{i}, \hat{b}_{i}, \hat{m}_{i}$ are respectively decay, blocking and matching rates obtained with the optimization model. In the Fig. 3 , the solution functions $\hat{o}_{1}(t), \hat{o}_{2}(t)$ and $\hat{o}_{3}(t)$ computed with Runge-Kutta fourth are represented by doted lines. In this example, these new parameters $\hat{p}_{1}, \hat{p}_{2}$ and $\hat{p}_{3}$ provide a quite good approximation. 


\subsection{Feasibility Tests}

Our feasibility tests follow roughly the procedure above:

1. Parameters $P$ are drawn at random,

2. solution functions $o_{i}(t)$ are computed by Runge-Kutta fourth method (precision of $1 \mathrm{E}-3)$,

3. from these solution functions, measures $M_{j}$ are extracted on all consecutive intervals $[a, b]$ from 0 such as $\int_{a}^{b} o_{i}(t) d t=1$ with $j \in \llbracket 1,4 \rrbracket$ and $b \leq b_{j}$,

4. the optimizer is launched with $M_{j}$ in the non-cognitive problem previously described,

5. new parameters $\hat{P}_{j}$ are then calculated by the optimizer,

6. solution functions $\hat{o}_{i}(t)$ from $\hat{P}_{j}$ are computed by Runge-Kutta fourth method (precision of 1E-3),

7. Errors $e_{j, l}$ are determinated with $l \in \llbracket 1,4 \rrbracket$ and $l \geq j$.

More precisely, four measure sets $M_{j}$ are respectively extracted on intervals $[0,5],[0,10],[0,15]$ and $[0,20](3)$. These measure sets simulate respectively the firs $5 \mathrm{~min}$, the firs $10 \mathrm{~min}$, the firs $15 \mathrm{~min}$ and the whole session. For each measure set, model parameters and solution functions are computed (4 and 5).

Our objective is to predict the ideas number in the future. Therefore, we do not calculate only an error on $M_{j}$ but also on next intervals. For example, for $M_{1}$ (interval $[0,5])$, four errors are calculated on intervals $[0,5],[0,10],[0,15]$ and $[0,20]$. In this way, errors give some information about the solution functions accuracy after the last measure (in the future).

We chose the following error calculus on interval $[a, b]$ : $e=\sum_{i=1}^{n}\left|\int_{a}^{b}\left(\hat{o}_{i}(t)-o_{i}(t)\right) d t\right|$, corresponding to the difference between the initial number of ideas and the optimized number of ideas for the group.

In our test, we vary the group size from 2 to 10 . For each size, the test procedure is repeated fi e times from which the mean, the variance, the minimum and the maximum of errors are calculated.

An extract of our tests results are presented in the Table 1. In the firs column, the group size is pointed out, the total solving time is given in the second column and, in the other columns, errors are calculated on considered intervals. Rows contain intervals on which measures have been extracted. These tests have been performed by GAMS/CONOPT3 non-linear optimizer ${ }^{4}$. We used default configuratio options of CONOPT3. The total solving time integrates the GAMS's compilation time and the CONOPT3's optimization time.

In the Table 1, solving time is described in the following format "minutes:seconds". On average, more the group size is important more the problem is difficul to solve. This tendency can be observed through the solving time. The solving time remains reasonable; its maximum value is $16 \mathrm{~s}$ for ten persons. As we can see, errors are not too different between measure intervals and extrapolated intervals. This seems to confir the adequacy of the proposed approach to the prediction of the ideas number in the

\footnotetext{
${ }^{4}$ On a PC computer (Pentium Dual-Core E5200 2.5GHZ and 4 GO RAM) whith Windows XP operating system.
} 
Table 1 Non-cognitive tests results

\begin{tabular}{|c|c|c|c|c|c|c|}
\hline Group size & Measures & Solving time & Error $[0,5]$ & Error $[0,10]$ & Error $[0,15]$ & Error $[0,20]$ \\
\hline \multirow[t]{13}{*}{2} & \multirow[t]{4}{*}[0,5]{} & Mean:0:1 & Mean: 0.043 & Mean: 0.660 & Mean: 2.188 & Mean: 4.155 \\
\hline & & Var: 0:1 & Var: 0.000 & Var: 0.133 & Var: 1.023 & Var: 3.195 \\
\hline & & Min: 0:1 & Min:0.012 & Min: 0.179 & Min: 0.794 & Min: 1.614 \\
\hline & & $\begin{array}{l}\text { Max: } 0: 1 \\
\text { Mean: } 0: 1\end{array}$ & Max: 0.069 & $\begin{array}{l}\text { Max: } 1.300 \\
\text { Mean: } 0.154\end{array}$ & $\begin{array}{l}\text { Max: } 3.910 \\
\text { Mean: } 0.270\end{array}$ & $\begin{array}{l}\text { Max: } 7.098 \\
\text { Mean: } 0.620\end{array}$ \\
\hline & \multirow{2}{*}[0,10]{} & Var: 0:1 & & Var: 0.003 & Var: 0.093 & Var: 0.422 \\
\hline & & Min: 0:1 & & Min: 0.047 & Min: 0.039 & Min: 0.106 \\
\hline & \multirow[t]{3}{*}[0,15]{} & $\begin{array}{l}\text { Max: 0:1 } \\
\text { Mean: 0:1 }\end{array}$ & & Max: 0.212 & $\begin{array}{l}\text { Max: } 0.864 \\
\text { Mean: } 0.303\end{array}$ & $\begin{array}{l}\text { Max: } 1.418 \\
\text { Mean: } 0.418\end{array}$ \\
\hline & & Var: 0:1 & & & Var: 0.016 & Var: 0.063 \\
\hline & & Min: 0:1 & & & Min: 0.088 & Min: 0.078 \\
\hline & \multirow[t]{4}{*}[0,20]{} & $\begin{array}{l}\text { Max: } 0: 1 \\
\text { Mean: } 0: 1\end{array}$ & & & $\begin{array}{l}\text { Max: } 0.463 \\
\text { Mean: } 0.479\end{array}$ & Max: 0.852 \\
\hline & & Var: 0:1 & & & & Var: 0.068 \\
\hline & & Min: 0:1 & & & & Min: 0.138 \\
\hline & & Max: 0:1 & & & & Max: 0.857 \\
\hline \multirow[t]{10}{*}{4} & \multirow[t]{3}{*}[0,5]{} & Mean: 0:1 & Mean: 1.415 & Mean: 2.119 & Mean: 2.328 & Mean: 2.380 \\
\hline & & Var: 0:1 & Var: 0.101 & Var: 0.343 & Var: 0.424 & Var: 0.458 \\
\hline & & Min: $0: 1$ & Min: 1.028 & Min: 1.352 & Min: 1.377 & Min: 1.372 \\
\hline & {$[0,10]$} & $\begin{array}{l}\text { Max: } 0: 1 \\
\text { Mean: } 0: 1 \\
\text { Var: } 0: 1 \\
\text { Min: } 0: 1 \\
\text { Max: } 0: 1 \\
\text { Mean: } 0: 1\end{array}$ & Max: 1.908 & $\begin{array}{l}\text { Max: } 2.881 \\
\text { Mean: } 2.271 \\
\text { Var: } 0.152 \\
\text { Min: } 1.418 \\
\text { Max: } 2.830\end{array}$ & $\begin{array}{l}\text { Max: } 3.171 \\
\text { Mean: } 2.570 \\
\text { Var: } 0.117 \\
\text { Min: } 2.199 \\
\text { Max: } 3.129 \\
\text { Mean: } 2.697\end{array}$ & $\begin{array}{l}\text { Max: } 3.249 \\
\text { Mean: } 2.668 \\
\text { Var: } 0.099 \\
\text { Min: } 2.356 \\
\text { Max: } 3.214 \\
\text { Mean: } 2.822\end{array}$ \\
\hline & \multirow{2}{*}[0,15]{} & Var: 0:1 & & & Var: 0.173 & Var: 0.165 \\
\hline & & Min: 0:1 & & & Min: 2.199 & Min: 2.370 \\
\hline & \multirow[t]{4}{*}[0,20]{} & $\begin{array}{l}\text { Max: 0:1 } \\
\text { Mean: 0:1 }\end{array}$ & & & Max: 3.365 & $\begin{array}{l}\text { Max: } 3.508 \\
\text { Mean: } 2.843\end{array}$ \\
\hline & & Var: 0:1 & & & & Var: 0.152 \\
\hline & & Min: 0:1 & & & & Min: 2.370 \\
\hline & & Max: 0:1 & & & & Max: 3.508 \\
\hline \multirow[t]{8}{*}{8} & \multirow[t]{4}{*}[0,5]{} & Mean: 0:2 & Mean: 3.442 & Mean: 4.165 & Mean: 4.365 & Mean: 4.413 \\
\hline & & Var: 0:1 & Var: 0.080 & Var: 0.110 & Var: 0.124 & Var: 0.126 \\
\hline & & Min: 0:1 & Min: 2.932 & Min: 3.547 & Min: 3.717 & Min: 3.756 \\
\hline & & Max: 0:2 & Max: 3.718 & Max: 4.530 & Max: 4.763 & Max: 4.806 \\
\hline & \multirow[t]{4}{*}[0,10]{} & Mean: 0:2 & & Mean: 4.218 & Mean: 4.455 & Mean: 4.520 \\
\hline & & Var: $0: 1$ & & Var: 0.087 & Var: 0.095 & Var: 0.100 \\
\hline & & Min: 0:2 & & Min: 3.656 & Min: 3.873 & Min: 3.928 \\
\hline & & Max: 0:3 & & Max: 4.524 & Max: 4.755 & Max: 4.798 \\
\hline
\end{tabular}


Table 1 continued

\begin{tabular}{|c|c|c|c|c|c|c|}
\hline Group size & Measures & Solving time & Error $[0,5]$ & Error $[0,10]$ & Error $[0,15]$ & Error $[0,20]$ \\
\hline & {$[0,15]$} & Mean: 0:2 & & & Mean: 4.311 & Mean: 4.358 \\
\hline & & Var: 0:1 & & & Var: 0.124 & Var: 0.135 \\
\hline & & Min: $0: 2$ & & & Min:3.667 & Min:3.698 \\
\hline & & Max: 0:2 & & & Max: 4.648 & Max: 4.753 \\
\hline & {$[0,20]$} & Mean: 0:2 & & & & Mean: 4.358 \\
\hline & & Var: 0:1 & & & & Var:0.135 \\
\hline & & Min: 0:2 & & & & Min: 3.698 \\
\hline & & Max: 0:2 & & & & Max: 4.753 \\
\hline \multirow[t]{16}{*}{10} & {$[0,5]$} & Mean: 0:3 & Mean: 4.921 & Mean: 5.302 & Mean: 5.384 & Mean: 5.401 \\
\hline & & Var: 0:3 & Var: 0.234 & Var: 0.376 & Var: 0.448 & Var: 0.473 \\
\hline & & Min: $0: 2$ & Min: 4.514 & Min: 4.626 & Min: 4.566 & Min: 4.529 \\
\hline & & Max: 0:6 & Max: 5.589 & Max: 6.070 & Max: 6.181 & Max: 6.200 \\
\hline & {$[0,10]$} & Mean: 0:4 & & Mean: 5.561 & Mean: 5.708 & Mean: 5.748 \\
\hline & & Var: 0:5 & & Var: 0.252 & Var: 0.263 & Var: 0.262 \\
\hline & & Min: 0:2 & & Min: 4.885 & Min: 4.974 & Min: 5.000 \\
\hline & & Max: 0:8 & & Max: 6.154 & Max: 6.331 & Max: 6.378 \\
\hline & {$[0,15]$} & Mean: 0:5 & & & Mean: 5.727 & Mean: 5.770 \\
\hline & & Var: 0:6 & & & Var: 0.255 & Var: 0.254 \\
\hline & & Min: $0: 2$ & & & Min: 4.974 & Min: 5.000 \\
\hline & & Max: 0:8 & & & Max: 6.331 & Max: 6.378 \\
\hline & {$[0,20]$} & Mean: 0:5 & & & & Mean: 5.770 \\
\hline & & Var: 0:6 & & & & Var: 0.254 \\
\hline & & Min: $0: 2$ & & & & Min: 5.000 \\
\hline & & Max: 0:8 & & & & Max: 6.378 \\
\hline
\end{tabular}

future. Let us note that our error calculus is a cumulative calculus, that is for two participants if individual errors were 0.5 then the group error would be 1 . Depending to the way of production duration is assessed; this error can overestimate (equals to 0 ) or underestimate (equals to 2) the real value. We intend to assess the production interval $[a, b]$ by considering that $a$ is the instant of the end of the last production and, $b$ is the instant of the end of the current production. In most cases, the expression time (speech time) is short in the interval $[a, b]$, our error calculus will thus overestimate the real $e$.

\subsection{Discussion}

Our approach presented earlier seems appropriate to predict ideas number using differential equations based on models. We believe that this approach can be useful in several other situations if we adapt the generic optimization model.

Some important advantages of this approach are: 
- model parameters can be calculated only with measures through the optimization model,

- the solving time of the optimization model can be fast,

- it can be used when the solution functions of differential equations are not known, or if they were known when the integral functions remain unknown,

- it processes cases where measures overlap.

Of course, this approach shows also some disadvantages:

- The numerical method provides an approximation of solution functions,

- the optimization model requires an hand configuratio by definin the precision ( $x_{i}$ points), the model part of the objective function and errors range.

- The Euler's method is very sensitive to the function variations inside define steps $\left(x_{i+1}-x_{i}\right)$. As the slope of the straight line is calculated only at the start of steps $\left(x_{i}\right)$, if the slope strongly varies (that is slope in others points is very different), then the Euler's linear approximation could be really wrong.

Our feasibility test confirm that the presented optimization model could be used to determine parameters of the non-cognitive model during a brainstorming session only with the knowledge of ideas production duration. Moreover, solution functions determined with these computed parameters allow the prediction of ideas number in the future (after the expression of the last idea).

\section{Cognitive Model of Brainstorming}

The cognitive model used in this part is more complex than the non-cognitive model (see Brown and Paulus 1996). We will show that this cognitive model is out of the applicability scope of the previous generic optimization model. A more accurate new model will be proposed to solve problems posed by the cognitive model. This general optimization model will be adapted to the cognitive model of brainstorming, and we will test its feasibility on artificia tests. As for the non-cognitive model, we will discuss the tests, advantages and disadvantages of this new optimization model.

\subsection{Mathematical Model}

In the cognitive model, three cognitive processes are introduced: idea generation, idea memory and idea output. This model assumes that a participant generates ideas (generation process) and stores them in short-term memory (memorization process) and then eventually extracts some of them from its short-term memory to express them (output process).

For each participant $i$ of a group of size $n$, this model is represented by the following three equations:

$$
\begin{aligned}
& \frac{d g_{i}(t)}{d t}=-a_{i} g_{i}(t)-b_{i} \sum_{j \neq i} o_{j}(t) \\
& \frac{d s_{i}(t)}{d t}=-c_{i} s_{i}(t)+g_{i}(t)-o_{i}(t) \text { with } g_{i}(t) \geq 0,0 \leq s_{i}(t) \leq K, o_{i}(t) \geq 0
\end{aligned}
$$




$$
\begin{gathered}
\text { and } t \in[0,+\infty[ \\
\frac{d o_{i}(t)}{d t}=-d_{i} o_{i}(t)+r_{i} s_{i}(t)-f_{i} \sum_{j \neq i} o_{j}(t)+m_{i}\left[\frac{1}{n-1} \sum_{j \neq i} o_{j}(t)-o_{i}(t)\right]
\end{gathered}
$$

where $g_{i}(t)$ describes the rate of generated ideas by the participant $i$ over time. it decays at the rate $a_{i}$. This model considers the produced ideas (outputs) of other participants block the ideas rate of $i$ at the rate $b_{i} . s_{i}(t)$ is the number of ideas in the short-term memory, $c_{i}$ is the forget rate. As for the non-cognitive model, $o_{i}(t)$ is the ideas rate which decay at the rate $d_{i} ; f_{i}$ and $m_{i}$ are respectively the blocking and the matching influences The additional term $r_{i}$ represents the rate of ideas extracted from the memory. In this modelling, ideas come from the idea generation process, and then are stored in memory (memorization process) and then are outputted (output process). Thus, at the session beginning $(t=0)$, the generation and memorization rates are equal to zero $\left(o_{i}(0)=s_{i}(0)=0\right)$.

As Coskun and Yilmaz (2009), to be more realistic, some physical constraints have been added. These constraints state that the rate of generated ideas $\left(g_{i}(t)\right)$, the rate of ideas in memory $\left(s_{i}(t)\right)$ and the rate of expressed ideas $\left(o_{i}(t)\right)$ must be positive or null. Moreover, the memory capacity is not considered infinit but is limited to the constant value $K$.

The impact of blocking factor on ideas number is formalized in the same manner as in non-cognitive model. Blocking influence both generation and output processes. In the generation process, ideas expressed by others are assumed to captivate a bit of attention of a participant and therefore may have the effect of inhibiting its train of thought. When someone speaks, usually the others will not have too much the desire to speak at the same time; this behavior is formalized by the blocking factor in the output stage. On the other hand, the matching only affects the output process and shares the same mathematical representation with the non-cognitive model.

For example, in the Fig. 4, the cognitive model is plotted for a group of two members. In this figure parameters vectors used are $p_{1}=(2.750,0,0,0.024,0.035,0.200$, $0.900,0.024,0.024,0.035)$ and $p_{1}=(2.250,0,0,0.024,0.035,0.200,0.900,0.024$, $0.024,0.035)$ where $p_{i}=\left(g_{i}(0), s_{i}(0), o_{i}(0), a_{i}, b_{i}, c_{i}, d_{i}, r_{i}, f_{i}, m_{i}\right)$ are the parameters vector of participant $i$ and $g_{i}(0)$ is the initial rate of ideas number, $a_{i}$ the decay rate and $b_{i}$ the blocking rate for the idea generation process; $s_{i}(0)$ always equal to zero is the initial rate of ideas number in memory and $c_{i}$ the forget rate; $o_{i}(0)$ also equal to zero is the initial rate of ideas number expressed, $d_{i}, r_{i}, f_{i}$ and $m_{i}$ are respectively the decay, extracted, blocking and matching rates of the output process.

The numerical Runge-Kutta method fourth $\operatorname{order}^{5}$ has been used to compute solution functions of each cognitive process. As for the non-cognitive model, due to model complexity, it is not always possible to fin solution functions in the form of analytical functions, then we will use numerical methods to approximate the above solution functions.

\footnotetext{
5 With a step of $1 \mathrm{E}-3$.
} 


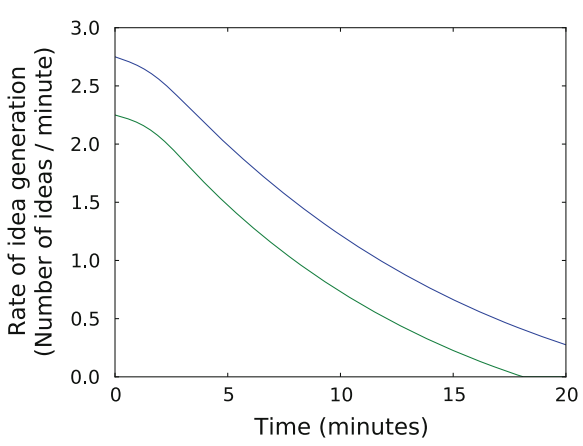

(a)

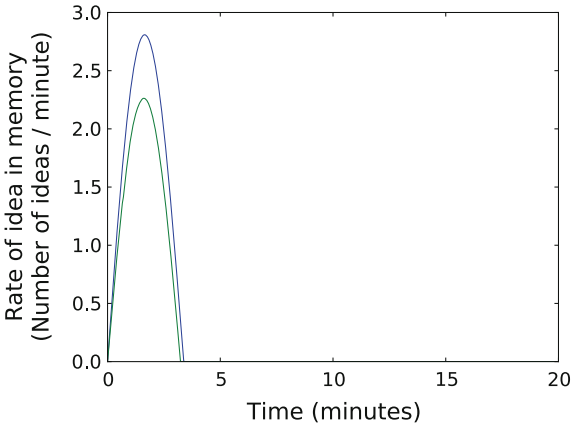

(b)

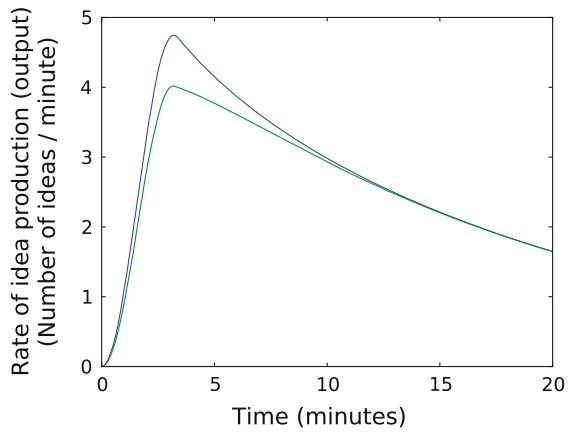

(c)

Fig. 4 Example of cognitive model with a group of two members. a Generation process. b Generation process. c Output process

\subsection{Runge-Kutta Based Generic Optimization Model for Parameters Calculation}

Unfortunately, in most cases, the previous generic optimization model based on Euler's method can not be used for the cognitive model. To illustrate this problem, let us consider the cognitive model presented in Fig. 5a. This model is the same as in Fig. 4 (same parameters vectors). Solution functions (full lines) are calculated by RungeKutta's fourth method with a step of 1E-3. From these functions, measure intervals $[a, b]$ corresponding to a surface of one $\left(\int_{a}^{b} o_{i}(t) d t=1\right)$ are extracted. As in our previous optimization model, Euler's method is applied on these measure intervals, thereby definin our Euler's solution functions (doted lines). As we can see in the Fig. 5a, the approximation caused by Euler's functions is too bad and will result in an infeasible optimization problem.

In this dynamic model, the variation of ideas rate inside measure intervals can be important. In this case, the Euler's method is not suitable (see the disadvantages previously discussed). One way to address this problem would be to increase the precision by decreasing the step. However, this approach entails an increase number of decision variables resulting in a more complex optimization model that will increase also the solving time. In the example of Fig. 5, a step of $0.5 \mathrm{E}-1$ can be used, nevertheless 

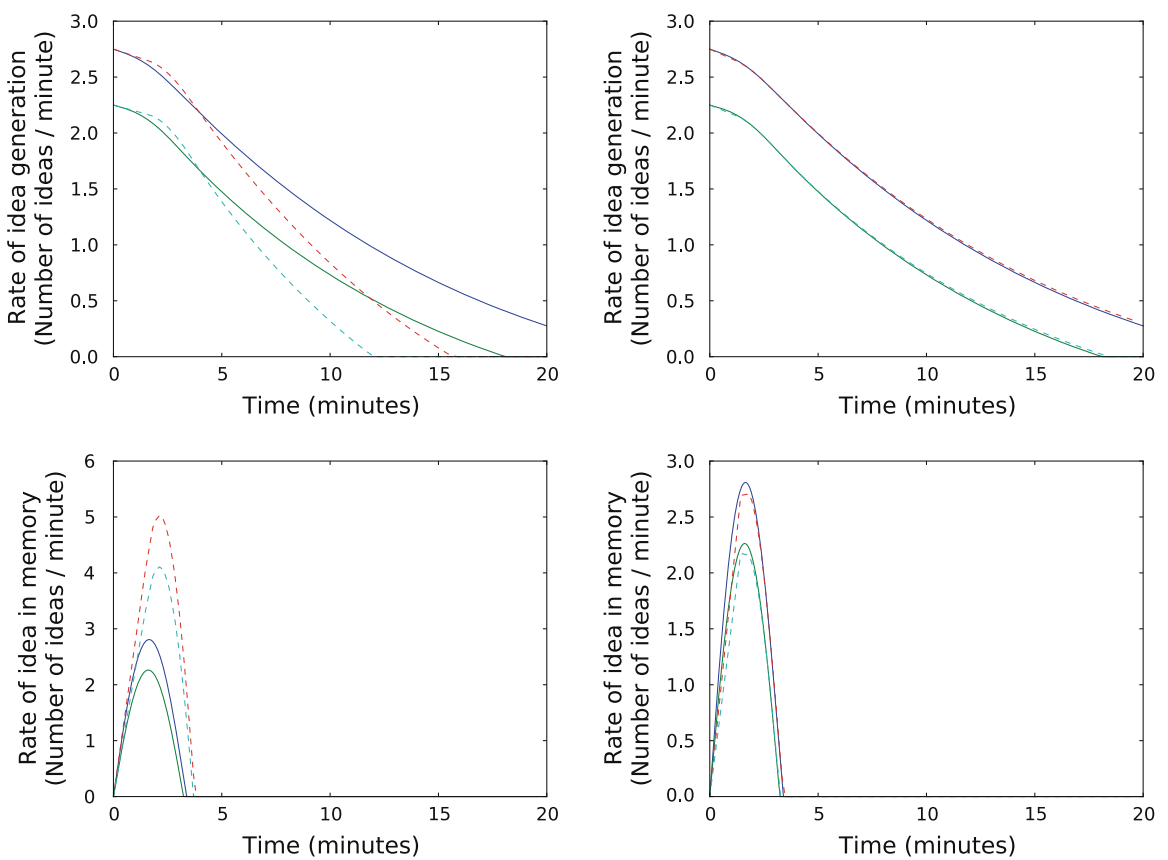

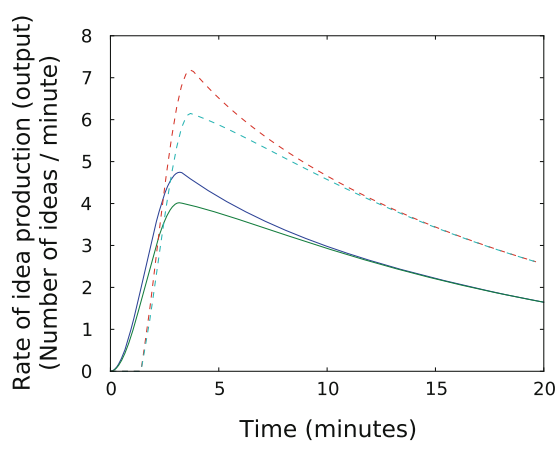

(a)

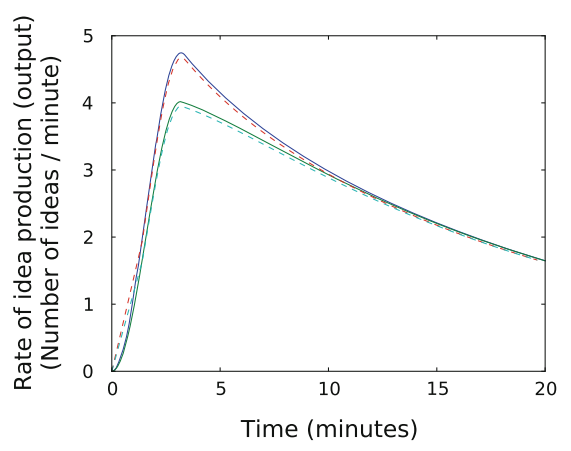

(b)

Fig. 5 Example of cognitive model with a group of two members. a Euler's method on measure intervals. b Runge-Kutta's fourth method on measure intervals

this would generate $73 \%$ more $x_{i}$ variables. Another way to deal with this problem would be to change the numerical method to a more precise. We chose this second approach by using the Runge-Kutta's fourth numerical method for solving firs order ordinary differential equations. As we can see in Fig. 5b, in the previous example, the Runge-kutta's method was applied to measure intervals and provides a satisfactory approximation.

The fourth order Runge-Kutta's method as Euler's method determines a linear approximation of solution functions of firs order differential equations. Let us consider the firs order differential equation $\frac{d y}{d x}=f(x, y)$ with initial value $y(a)=y_{o}$ 
and $\mathrm{f}(\mathrm{x}, \mathrm{y})$ is a known function define on an interval $\mathrm{I}=[a, b]$. Let $h=\frac{b-a}{n}$ be a real positive number where $n$ is the number of considered points. The real $h$ is called "step" and it is the step sizes between the points $x_{0}, x_{1}, \ldots, x_{n}$.

The forth order Runge-Kutta's method considers two sequences of real numbers $x_{i}$ and $y_{i}$ for all $i \in \llbracket 0, n-1 \rrbracket$ such that $x_{i+1}=x_{i}+h$ and $y_{i+1}=y_{i}+h K_{i}$ with $K_{i}=\frac{k_{i}^{1}+2 k_{i}^{2}+2 k_{i}^{3}+k_{i}^{4}}{6}$ where:

$$
\begin{aligned}
k_{i}^{1}= & f\left(x_{i}, y_{i}\right), \\
k_{i}^{2}= & f\left(x_{i}+\frac{h}{2}, y_{i}+\frac{h}{2} k_{i}^{1}\right), \\
& \text { using } k_{i}^{1} \text { as slope, } \\
k_{i}^{3}= & f\left(x_{i}+\frac{h}{2}, y_{i} \text { is the slope at } x_{i}\right. \text { (interval beginning), } \\
& \text { using } k_{i}^{2} \text { as slope }, \\
k_{i}^{4}= & f\left(x_{i}+h, y_{i}+h k_{i}^{3}\right), \quad k_{i}^{3} \text { is the slope at the interval middle } \\
& \text { using } k_{i}^{3} \text { as slope. }
\end{aligned}
$$

The values in initial condition are respectively $x_{0}=a$ and $y_{0}=y(a)$. The linear approximation function of $y(x)$ on $I$ is given by $\phi(x)=K_{i} x+y_{i}-K_{i} x_{i}$ for all $x \in\left[x_{i}, x_{i+1}\right]$ and $\phi\left(x_{0}\right)=y_{0}$. In the paper, when we will mention Runge-Kutta's method, we will refer to the fourth order Runge-Kutta's method.

To fin parameters of the cognitive model from measures $M_{a, b, i}=\int_{a}^{b} y(t) d t$ on $[a, b]$, we propose the following optimization model based on Runge-Kutta's numerical method:

\begin{tabular}{|l|l|}
\hline $\begin{array}{l}\text { Objective } \\
\text { function: }\end{array}$ & $\operatorname{Min}\left(\alpha \sum_{i=1}^{m} e_{i}^{2}+f_{d m}(p)\right)$ where $m$ is the number of measures, \\
Constraints: & $h_{i}=\left(x_{i+1}-x_{i}\right)$ for all $i \in \llbracket 0, m-1 \rrbracket$, \\
& $y_{i+1}=y_{i}+h_{i} K_{i}$ for all $i \in \llbracket 0, m-1 \rrbracket$, \\
& $K_{i}=\frac{k_{i}^{1}+2 k_{i}^{2}+2 k_{i}^{3}+k_{i}^{4}}{6}$ for all $i \in \llbracket 0, m-1 \rrbracket$, \\
& $k_{i}^{1}=f\left(x_{i}, y_{i}, p\right)$ for all $i \in \llbracket 0, m-1 \rrbracket$, \\
& $k_{i}^{2}=f\left(x_{i}+\frac{h_{i}}{2}, y_{i}+\frac{h_{i}}{2} k_{i}^{1}, p\right)$ for all $i \in \llbracket 0, m-1 \rrbracket$, \\
& $k_{i}^{3}=f\left(x_{i}+\frac{h_{i}}{2}, y_{i}+\frac{h_{i}}{2} k_{i}^{2}, p\right)$ for all $i \in \llbracket 0, m-1 \rrbracket$, \\
& $k_{i}^{4}=f\left(x_{i}+h_{i}, y_{i}+h_{i} k_{i}^{3}, p\right)$ for all $i \in \llbracket 0, m-1 \rrbracket$, \\
& $S_{i+1}=\frac{h_{i}\left(y_{i}+y_{i+1}\right)}{2}$ for all $i \in \llbracket 0, m-1 \rrbracket$, \\
& $M_{a, b, i}=\sum_{j=o r d(a)} S_{j}+e_{i}$ for all $i \in \llbracket 1, m \rrbracket$, \\
& \\
& $M_{a, b, i}$ is the $i$ th measure on $[a, b]$, \\
& $x_{i}$ sorted abcissa of considered points, \\
& $h_{i}$ the step, \\
& $\alpha$ errors weight, \\
& ord $(u)$ returns the position of $u$ in $x$,
\end{tabular}




\begin{tabular}{|l|l|}
\hline Decision & $f$ differential equation $\frac{d y}{d x}=f(x, y, p)$ of the model, \\
& \\
& $p$ parameters in differential equation, \\
& $K_{i}, k_{i}^{1}, k_{i}^{2}, k_{i}^{3}$ and $k_{i}^{4}$ are Runge-Kutta's slopes, \\
& $y_{i}$ points determined by the Runge-Kutta's linear approximation, \\
& $S_{i}$ trapezoid area with height $x_{i+1}-x_{i}$, \\
& $f_{d m}$ objective function related to differential equation, \\
& $e_{i}$ error of the $i$ th measure. \\
\hline
\end{tabular}

In this new optimization model, only the numerical method has been changed. As Runge-Kutta's and Euler's methods provide a linear approximation, the optimization model is identical to the Euler based optimization model presented previously, except that Euler's slopes are replaced by Runge-Kutta's slopes.

\subsection{Optimization Model for Parameters Calculation of Cognitive Model}

To determine parameters of the cognitive model, the Runge-Kutta based generic optimization model is applied. We used the same compromise between precision and solving time by choosing as Runge-Kutta's points the start and the end of measure intervals. Moreover, the interval measure extremities are determined in the way that the number of ideas for one participant is equal to 1 (that is $\int_{a}^{b} o_{i}(t) d t=1$ ).

The optimization model can be described as following:

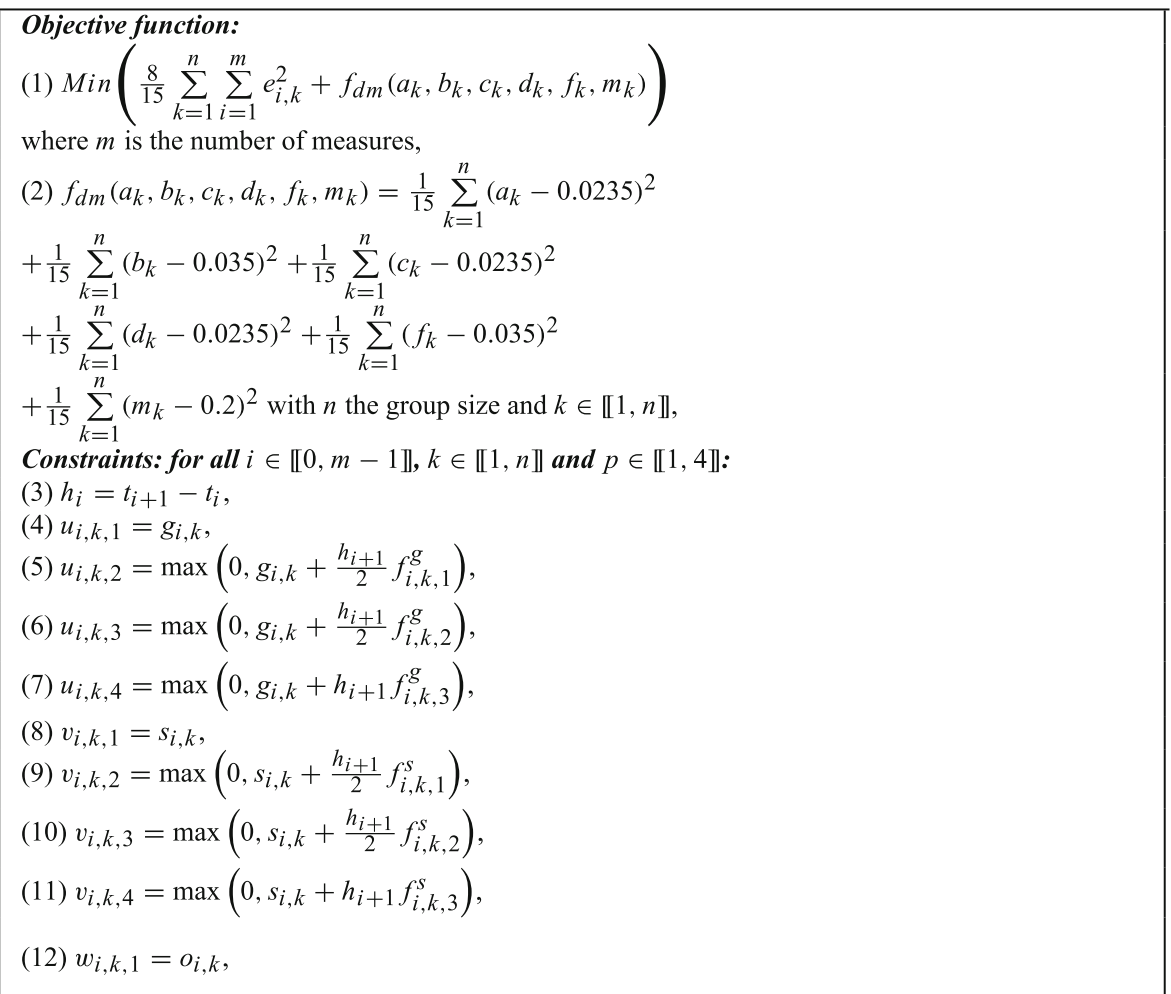




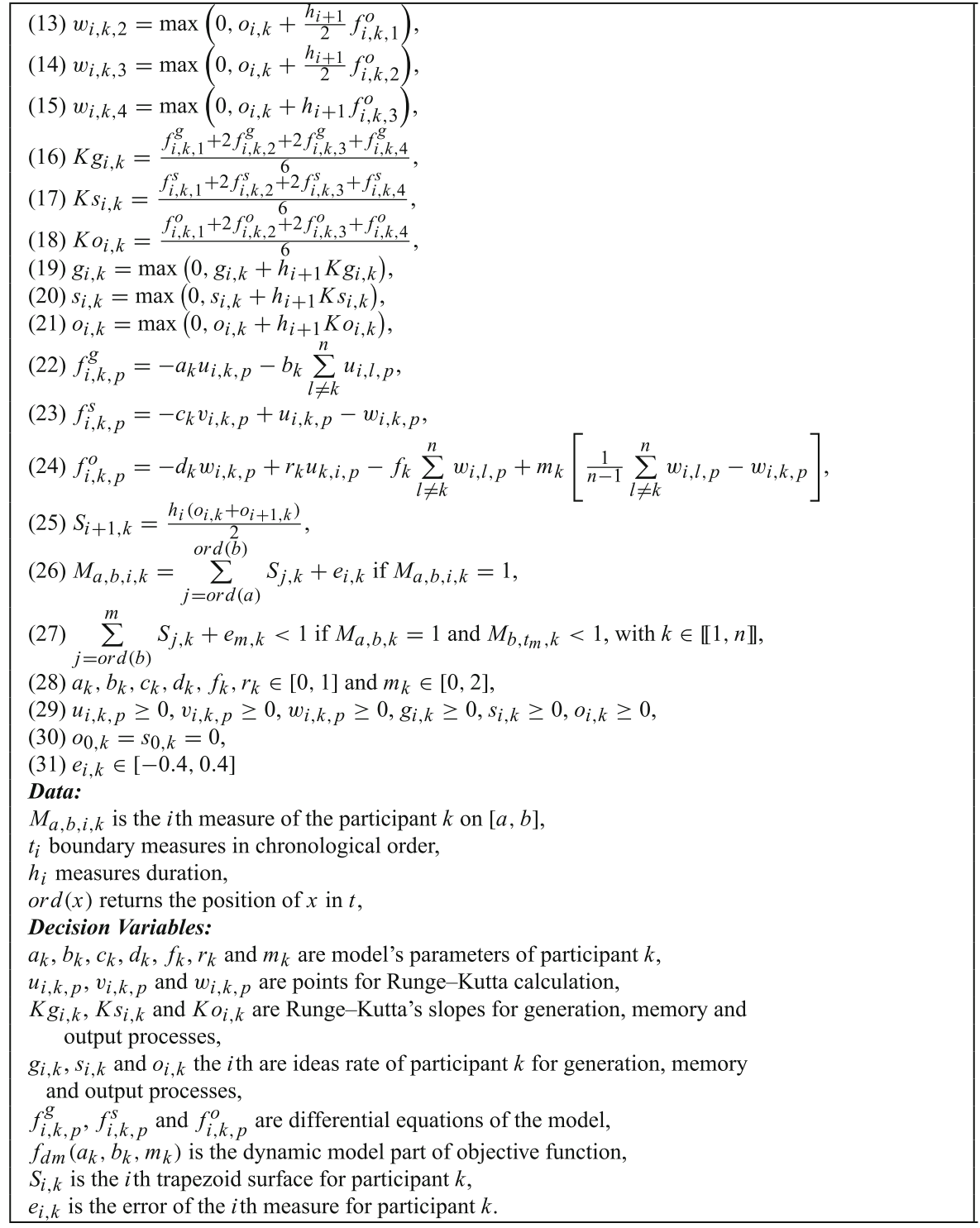

As for the non-cognitive problem, objective function minimizes errors (1) and the distance to reference values (2). The reference values used are those that have been established by Coskun and Yilmaz (2009). In this objective function, the minimization of errors (coefficien of $\frac{8}{15}$ ) is preferred to the minimization of distances between factors and their reference values (coefficien of $\frac{1}{15}$ ). In our notation, $g, s$ and $o$ mean that the designated variables refer to the generation, memorization and output processes. The constraints between (4) and (24) describe the application of the Runge-Kutta method for these processes. The variables $f_{i, k, 1}^{\mathrm{g}}\left(\right.$ or $f_{i, k, 1}^{\mathrm{s}}, f_{i, k, 1}^{\mathrm{o}}$ ) correspond to $k^{1}$ Runge-Kutta's slopes, $f_{i, k, 3}^{\mathrm{g}}\left(\right.$ or $\left.f_{i, k, 2}^{\mathrm{s}}, f_{i, k, 2}^{\mathrm{o}}\right)$ to $k^{2}, f_{i, k, 3}^{\mathrm{g}}\left(\right.$ or $\left.f_{i, k, 3}^{\mathrm{s}}, f_{i, k, 3}^{\mathrm{o}}\right)$ to $k^{3}$ and 


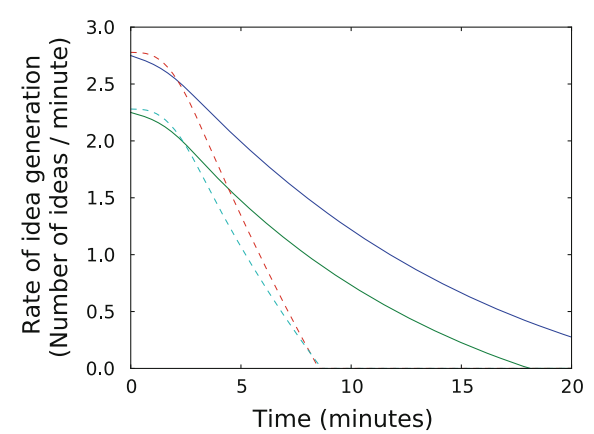

(a)

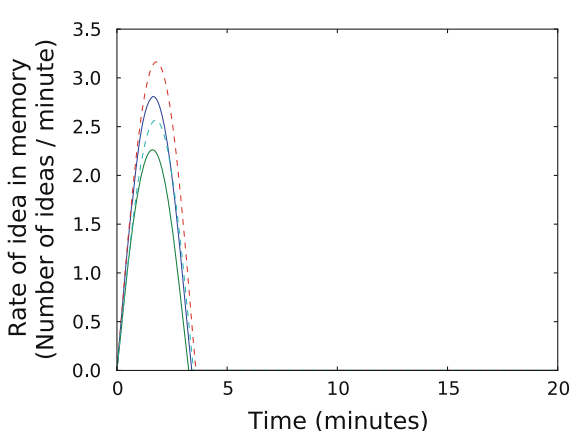

(b)

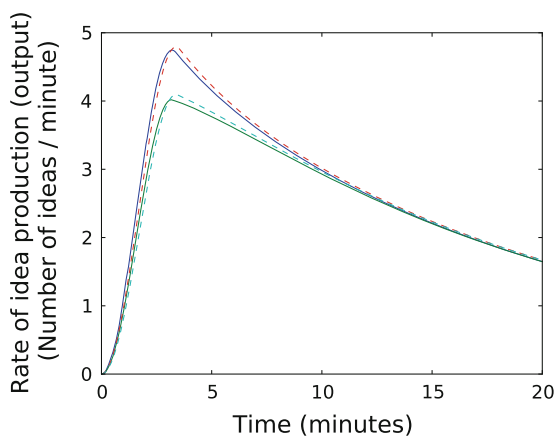

(c)

Fig. 6 Cognitive model with initial and found by optimization parameters. a Generation process. b Memorization process. c Output process

$f_{i, k, 4}^{\mathrm{g} \text { or } \mathrm{s} \text { or o }}$ to $k^{4}$. For the generation process, the variables $u_{i, k, 1}$ represent the variables $g_{i, k}, u_{i, k, 2}$ the variables $g_{i, k}+\frac{h_{i+1}}{2} f_{i, k, 1}^{g}, u_{i, k, 3}$ the variables $g_{i, k}+\frac{h_{i+1}}{2} f_{i, k, 2}^{g}$ and $u_{i, k, 3}$ the variables $g_{i, k}+h_{i+1} f_{i, k, 3}^{g}$. In that way, $f_{i, k, 1}^{\mathrm{g}}$ represent $f\left(g_{i, k}\right), f_{i, k, 2}^{\mathrm{g}}$ represent $f\left(g_{i, k}+\frac{h_{i+1}}{2} k^{1}\right)$, and so on. Let us note that $u_{i, k, p}$ are positive or null (max function). The variables $K g_{i, k}$ are the fina slopes of Runge-Kutta's method. The same formalization is used for other processes, and the rest of constraints are identical to the non-cognitive problem.

We applied the optimization model described above on measures extracted from the model of the Fig. 4. The parameters obtained by the optimizer are : $\hat{p}_{1}=$ $(2.778,0,0,0,0.108,0,0.037,0.772,0.021,0.227)$ and $\hat{p}_{2}=(2.280,0,0,0,0.078$, $0,0.011,0.766,0.047,0.176)$. In the Fig. 6 , initial and computed by optimization solution functions are respectively plotted in full lines and in dotted lines ${ }^{6}$. As we can see in Fig. 6, in the output process, the approximation provided is quite good. In many cases, many solutions functions exist for the same output solution functions; which ex-

6 The initial model uses $p_{1}$ and $p_{2}$ parameters and the computed by optimization model $\hat{p}_{1}$ and $\hat{p}_{2}$. In both models, solution functions are calculated by fourth order Runge-Kutta's method with a step of 1E-3 
plains why in the example of the Fig. 6, the generation and the memorization solution functions are quite different to the initial ones.

\subsection{Feasibility Tests}

The proposed feasibility tests follow the same procedure than those of the non-cognitive model. Parameters are drawn at random and some measures are extracted. From these measures, the Runge-Kutta based optimization model is applied, providing new parameters and an error is calculated. Measures are extracted on four intervals $([0,5]$, $[0,10],[0,15]$ and $[0,20])$, and errors are determined on these four intervals allowing us to test predictions. For each size, the procedure is repeated fi e times.

An extract of our tests results are presented in the Table 2. These tests have been performed by GAMS/CONOPT3 non-linear optimizer ${ }^{7}$. In our use context, the solving process must not take too much time, we thus choose to limit the optimization time to $1.30 \mathrm{~min}$ (set in configuratio options of CONOPT3). As previously, the total solving time integrates the GAMS's compilation time and the CONOPT3's optimization time.

Let us note that for groups of 8 and 9 members, the solving time exceeds the limit of 1.30 minutes: this is due to the GAMS compilation time. In addition, for these groups, the optimizer did not complete its optimization process, and therefore did not provide the locally optimal solution but only a feasible solution. This explains why the solving time is approximately constant (about $1.35 \mathrm{~min}$ ).

The errors are not too different between measure intervals and extrapolated intervals, which seems to confir the adequacy of the Rung-Kutta based optimization model to our prediction context. Moreover, this optimization model can be directly applied to groups of small size (from 2 to 6). However, it requires further developments to reduce the solving time for groups larger than 6 . In our tests, the solving time exceeds the limit of $1.30 \mathrm{~min}$ for groups of 10 participants.

\subsection{Discussion}

The presented fourth order Runge-Kutta based optimization model can be used when Euler's method is not enough precise and could result to an infeasible optimization problem. This method generally increases the precision but also the solving time. It shares many advantages and disadvantages with Euler's based problem, which are:

- Advantages:

- Model parameters can be calculated only from measures,

- The solving can be even fast (as for small groups in our tests),

- It can be used when solution functions of differential equations are unknown, or when their integrals are also not known,

- It processes cases where measures overlap,

- It is more precise than Euler's based optimization model.

\footnotetext{
7 On a PC computer (Pentium Dual-Core E5200 2.5GHZ and 4 GO RAM) with Windows XP operating system.
} 
Table 2 Cognitive tests results

\begin{tabular}{|c|c|c|c|c|c|c|}
\hline Group size & Measures & Solving time & Error $[0,5]$ & Error $[0,10]$ & Error $[0,15]$ & Error $[0,20]$ \\
\hline \multirow[t]{14}{*}{2} & \multirow[t]{4}{*}[0,5]{} & Mean:0:2 & Mean: 0.282 & Mean: 0.579 & Mean: 1.767 & Mean: 3.544 \\
\hline & & Var: 0:1 & Var: 0.008 & Var: 0.128 & Var: 0.876 & Var: 2.443 \\
\hline & & Min: 0:1 & Min:0.199 & Min: 0.114 & Min: 0.722 & Min: 1.117 \\
\hline & & Max: 0:3 & Max: 0.439 & Max: 1.020 & Max: 2.983 & Max: 5.522 \\
\hline & \multirow[t]{3}{*}[0,10]{} & Mean: 0:3 & & Mean: 0.111 & Mean: 0.209 & Mean: 0.838 \\
\hline & & Var: 0:4 & & Var: 0.005 & Var: 0.019 & Var: 0.942 \\
\hline & & Min: 0:1 & & Min: 0.030 & Min: 0.040 & Min: 0.182 \\
\hline & & $\begin{array}{l}\text { Max: 0:6 } \\
\text { Mean: 0:5 }\end{array}$ & & Max: 0.191 & $\begin{array}{l}\text { Max: } 0.452 \\
\text { Mean: } 0.303\end{array}$ & $\begin{array}{l}\text { Max: } 2.749 \\
\text { Mean: } 0.778\end{array}$ \\
\hline & \multirow{2}{*}[0,15]{} & Var: 0:9 & & & Var: 0.028 & Var: 0.406 \\
\hline & & Min: 0:1 & & & Min: 0.019 & Min: 0.087 \\
\hline & \multirow[t]{4}{*}[0,20]{} & $\begin{array}{l}\text { Max: 0:9 } \\
\text { Mean: 0:8 }\end{array}$ & & & $\begin{array}{l}\text { Max: } 0.506 \\
\text { Mean: } 0.442\end{array}$ & Max: 1.971 \\
\hline & & Var: 0:22 & & & & Var: 0.054 \\
\hline & & Min: 0:3 & & & & Min: 0.031 \\
\hline & & Max: 0:14 & & & & Max: 0.713 \\
\hline \multirow[t]{16}{*}{4} & \multirow[t]{4}{*}[0,5]{} & Mean: 0:23 & Mean: 0.543 & Mean: 0.950 & Mean: 5.182 & Mean: 9.763 \\
\hline & & Var: 0:58 & Var: 0.017 & Var: 0.351 & Var: 8.295 & Var: 24.326 \\
\hline & & Min: 0:18 & Min: 0.350 & Min: 0.309 & Min: 0.061 & Min: 0.520 \\
\hline & & Max: 0:38 & Max: 0.674 & Max: 1.819 & Max: 8.467 & Max: 15.062 \\
\hline & \multirow[t]{4}{*}[0,10]{} & Mean: 0:37 & & Mean: 0.485 & Mean: 4.104 & Mean: 8.622 \\
\hline & & Var: 3:2 & & Var: 0.132 & Var: 4.807 & Var: 22.979 \\
\hline & & Min: 0:19 & & Min: 0.004 & Min: 1.840 & Min: 3.020 \\
\hline & & Max: 0:50 & & Max: 0.933 & Max: 6.700 & Max: 15.464 \\
\hline & \multirow[t]{4}{*}[0,15]{} & Mean: 0:45 & & & Mean: 0.371 & Mean: 1.700 \\
\hline & & Var: 3:44 & & & Var: 0.043 & Var: 0.818 \\
\hline & & Min: 0:25 & & & Min: 0.141 & Min: 0.361 \\
\hline & & Max: 1:12 & & & Max: 0.755 & Max: 3.072 \\
\hline & \multirow[t]{4}{*}[0,20]{} & Mean: 1:15 & & & & Mean: 0.418 \\
\hline & & Var: 2:37 & & & & Var: 0.012 \\
\hline & & Min: 1:0 & & & & Min: 0.207 \\
\hline & & Max: 1:35 & & & & Max: 0.507 \\
\hline \multirow[t]{8}{*}{8} & \multirow[t]{4}{*}[0,5]{} & Mean: 1:30 & Mean: 0.804 & Mean: 3.937 & Mean: 5.139 & Mean: 5.409 \\
\hline & & Var: 1:39 & Var: 0.766 & Var: 3.893 & Var: 3.568 & Var: 2.609 \\
\hline & & Min: $1: 10$ & Min: 0.004 & Min: 0.698 & Min: 2.046 & Min: 2.949 \\
\hline & & Max: 1:35 & Max: 2.420 & Max: 6.685 & Max: 7.103 & Max: 7.459 \\
\hline & \multirow[t]{4}{*}[0,10]{} & Mean: 1:35 & & Mean: 1.831 & Mean: 2.039 & Mean: 2.357 \\
\hline & & Var: 0:1 & & Var: 0.914 & Var: 3.453 & Var: 5.760 \\
\hline & & Min: 1:34 & & Min: 0.936 & Min: 0.787 & Min: 0.508 \\
\hline & & Max: 1:36 & & Max: 3.566 & Max: 5.719 & Max: 6.906 \\
\hline
\end{tabular}


Table 2 continued

\begin{tabular}{|c|c|c|c|c|c|c|}
\hline Group size & Measures & Solving time & Error $[0,5]$ & Error $[0,10]$ & Error $[0,15]$ & Error $[0,20]$ \\
\hline & {$[0,15]$} & Mean: 1:35 & & & Mean: 4.715 & Mean: 5.779 \\
\hline & & Var: 0:1 & & & Var: 37.694 & Var: 75.011 \\
\hline & & Min: 1:35 & & & Min:0.593 & Min:0.187 \\
\hline & & Max: 1:36 & & & Max: 16.866 & Max: 22.984 \\
\hline & {$[0,20]$} & Mean: 1:35 & & & & Mean: 3.009 \\
\hline & & Var: $0: 1$ & & & & Var:16.500 \\
\hline & & Min: $1: 35$ & & & & Min: 0.207 \\
\hline & & Max: 1:35 & & & & Max: 10.895 \\
\hline \multirow[t]{16}{*}{9} & {$[0,5]$} & Mean: 1:35 & Mean: 1.090 & Mean: 2.956 & Mean: 2.844 & Mean: 2.864 \\
\hline & & Var: 0:1 & Var: 0.469 & Var: 7.034 & Var: 6.319 & Var: 4.475 \\
\hline & & Min: $1: 35$ & Min: 0.490 & Min: 0.293 & Min: 0.191 & Min: 0.134 \\
\hline & & Max: 1:35 & Max: 2.351 & Max: 7.628 & Max: 6.971 & Max: 6.413 \\
\hline & {$[0,10]$} & Mean: 1:35 & & Mean: 1.112 & Mean: 1.125 & Mean: 1.352 \\
\hline & & Var: 0:1 & & Var: 1.209 & Var: 0.624 & Var: 1.460 \\
\hline & & Min: 1:35 & & Min: 0.065 & Min: 0.164 & Min: 0.319 \\
\hline & & Max: 1:36 & & Max: 2.746 & Max: 1.989 & Max: 3.652 \\
\hline & {$[0,15]$} & Mean: 1:36 & & & Mean: 3.293 & Mean: 4.098 \\
\hline & & Var: 0:1 & & & Var: 16.018 & Var: 24.401 \\
\hline & & Min: $1: 35$ & & & Min: 0.090 & Min: 0.180 \\
\hline & & Max: 1:38 & & & Max: 10.968 & Max: 13.750 \\
\hline & {$[0,20]$} & Mean: 1:36 & & & & Mean: 4.358 \\
\hline & & Var: $0: 1$ & & & & Var: 31.961 \\
\hline & & Min: $1: 35$ & & & & Min: 0.004 \\
\hline & & Max: 1:36 & & & & Max: 15.427 \\
\hline
\end{tabular}

- Disadvantages:

- Even if the model is more precise, Runge-Kutta's method provides an approximation of solution functions,

- The optimization model requires an manual configuration

- As Euler's method, Runge-kutta's method is also sensitive of function variations inside steps $\left(x_{i+1}-x_{i}\right)$.

In our application context, we intend to determine parameters of dynamic models during the brainstorming session with the presented optimization models. Thanks to these calculated parameters, we will be able to predict the number of ideas in the session future. Therefore, it does not necessarily need to compute new parameters after each measure (idea). Indeed, if current measures well fi to the solutions functions determined before with previous ideas, it is not required to reapply the proposed optimization models.

In their experimentations, Coskun and Yilmaz (2009) noted that in the firs half of the brainstorming session, the non-cognitive model fit better to the number of ideas 
recorded than the cognitive model, and conversely, the cognitive model was better in the second half of the session. Therefore, the use of the both models (non-cognitive and cognitive) and our both optimization models (Euler based and Runge-Kutta based) could be interesting for the prediction of ideas number.

\section{Conclusion}

In this paper, we presented an approach allowing the prediction of ideas number during a brainstorming session. This prediction is based on two dynamic models of brainstorming, the non-cognitive and the cognitive models proposed by Brown and Paulus (1996), and extended by Coskun and Yilmaz (2009). These models describes for each participant, the evolution of ideas number over time according to three social factors (decay, blocking and matching), and are formalized by differential equations. The solution functions of these models offer a precise description of ideas number over time. Through them, it is then possible to calculate the number of ideas of each participant on any time intervals and thus in the future ${ }^{8}$. However, to be able to compute solution functions, it is necessary to determine the parameters of these models.

In many dynamic models, analytical solution functions or their integrals are unknown. In our approach, we propose to use optimization model for model parameters calculation in which solution functions are approximated by numerical methods. In our context of prediction, we want to determine models' parameters in real-time (that is during the brainstorming session), therefore the time of parameters calculation is very important and must be reasonable.

We developed a generic optimization model based on Euler's numerical method and we applied it to the non-cognitive model. Through artificia tests, this optimization model appears appropriate to the parameters calculation of the non-cognitive, and satisfie our time constraint. Moreover, these tests confir the adequacy of the proposed approach to predict the ideas number in the future. However, the Euler based generic optimization model can not be applied to the cognitive model on measure intervals. We then proposed the use of a more precise numerical method, the forth order Runge-Kutta's method. A new generic optimization model based on it for parameters calculation was presented, and we applied it to the cognitive model. Our tests showed the adequacy of this new optimization model to our prediction context. This optimization model can be directly applied to groups of small size (from 2 to 6), however requires further developments to reduce parameters calculation time for groups larger than 6.

We believe that our approach for determining parameters of dynamic models from measurements can be useful in many other situations, either by adapting the generic optimization models proposed or by changing the numerical method. Some advantages of this approach are: models parameters can be calculating only from measures, the parameters calculation time may be short, it can be used when solution functions or their integral functions are not known, the measures can overlap, and the change of numerical method is relatively easy. Some disadvantages are: numerical methods

8 Which we call prediction. 
provide an approximation of solution functions, the optimization model requires hand configuration and the proposed numerical methods are sensitive to the function variations inside steps.

This approach will be tested on face to face real brainstorming sessions by using microphones to measure the duration of ideas production, and in electronic brainstorming sessions by using a timer. Through these experimentations, it might be possible to study in more details the eventual dynamic of the social factors (parameterization of the dynamic models). We also plan to extend this work to ideas quality by adapting it to the Bounded Ideation Theory proposed by Briggs and Reinig (2010). Finally, the proposed predictions will be integrated to our efficien y measure of decision making meetings (see Camilleri and Zarat 2009) and will contribute to our GDSS facilitation tool.

\section{References}

Adla A, Zaraté P, Soubie JL (2011) A proposal of toolkit for GDSS facilitators. Group Decis Negot 20(1):57-77

Ait-Haddou H, Camilleri G, Zaraté P (2010) Parameters calculation of brainstorming dynamic models. In: De Vreede GJ (ed) Group decision and negotiation (GDN). Center for Collaboration Science, pp 24-26

Borgatta EF, Bales RF (1953) Interaction of individuals in reconstituted groups. Sociometry 16(4):302-320

Bostrom RP, Anson R, Clawson VK (1993) Group facilitation and group support systems. Group support systems: new perspectives, pp 146-168

Briggs RO, Kolfschoten GL, de Vreede GJ, Albrecht CC, Lukosch SG (2010) Facilitator in a box: computer assisted collaboration engineering and process support systems for rapid development of collaborative applications for high-value tasks. In: HICSS, IEEE Computer Society, pp 1-10

Briggs RO, Reinig BA (2010) Bounded ideation theory. J Manag Inf Syst 27(1):123-144

Brown V, Paulus PB (1996) A simple dynamic model of social factors in group brainstorming. Small Group Res 27(1):91-114

Brown VR, Paulus PB (2002) Making group brainstorming more effective: recommendations from an associative memory perspective. Curr Dir Psychol Sci 11(5):208-212

Camacho LM, Paulus PB (1995) The role of social anxiousness in group brainstorming. J Pers Soc Psychol 68(6):1071-1080

Camilleri G, Zaraté P (2009) Towards a dynamic estimation of collective decision-making meetings effi ciency. In: Kilgour M, Wang Q (eds) Group decision and negotiation (GDN), Institute for Operations Research and the Management Sciences (INFORMS), pp 27-29

Coskun H (2005) Cognitive stimulation with convergent and divergent thinking exercises in brainwriting. Small Group Res 36(4):466-498

Coskun H, Yilmaz O (2009) A new dynamical model of brainstorming: linear, nonlinear, continuous (simultaneous) and impulsive (sequential) cases. J Math Psychol 53(4):253-264

de Vreede GJ, Vogel DR, Kolfschoten GL, Wien J (2003) Fifteen years of gss in the field a comparison across time and national boundaries. In: HICSS, p 9

den Hengst M, Adkins M (2007) Which collaboration patterns are most challenging: a global survey of facilitators. In: HICSS, IEEE Computer Society, p 17

DeRosa DM, Smith CL, Hantula DA (2007) The medium matters: mining the long-promised merit of group interaction in creative idea generation tasks in a meta-analysis of the electronic group brainstorming literature. Comput Hum Behav 23(3):1549-1581. (Including the special issue: avoiding simplicity, confronting complexity: advances in designing powerful electronic learning environments)

DeSanctis G, Poole MS, Zigurs I (2008) The minnesota gdss research project: group support systems, group processes, and outcomes. J AIS 9(10), 551-608

Diehl M, Stroebe W (1987) Productivity loss in brainstorming groups: toward the solution of a riddle. J Pers Soc Psychol 53(3):497-509 
Diehl M, Stroebe W (1991) Productivity loss in idea-generating groups: tracking down the blocking effect. J Pers Soc Psychol 61(3):392-403

Hayne SC (1999) The facilitators perspective on meetings and implications for group support systems design. DATA BASE 30(3-4):72-91

Isaksen SG (1998) A review of brainstorming research: six critical issues for research. Monograph 302, Creative Problem Solving Group, Buffalo

Karau SJ, Williams KD (1993) Social loafing: a meta-analytic review and theoretical integration. J Pers Soc Psychol 65(4):681-706

Limayem M (2006) Human versus automated facilitation in the gss context. SIGMIS Database 37(2-3): $156-166$

Limayem M, DeSanctis G (2000) Providing decisional guidance for multicriteria decision making in groups. Inf Syst Res 11(4):386-401

Macaulay LA, Alabdulkarim A (2005) Facilitation of e-meetings: State-of-the-art review. In: EEE, IEEE Computer Society, pp 728-735

Mullen B, Johnson C, Salas E (1991) Productivity loss in brainstorming groups: a meta-analytic integration. Basic Appl Soc Psychol 12(1):3-23

Nijstad BA, Stroebe W, Lodewijkx HFM (2003) Production blocking and idea generation: does blocking interfere with cognitive processes?. J Exp Soc Psychol 39(6):531-548

Nunamaker JF, Dennis AR, Valacich JS, Vogel D, George JF (1991) Electronic meeting systems. Commun ACM 34(7):40-61

Nunamaker JF, Zhao JL, Briggs RO (2002) Intelligent workfl w techniques for distributed group facilitation. In: HICSS, p 42

Osborn AF (1957) Applied imagination: principles and procedures of creative problem-solving. Charles Scribner's Sons, New York

Oxley NL, Dzindolet MT, Paulus PB (1996) The effects of facilitators on the performance of brainstorming groups. J Soc Behav Pers 11:633-646

Paulus PB, Dzindolet MT (1993) Social influenc processes in group brainstorming. J Pers Soc Psychol 64(4):575-586

Schwarz R (1994) The skilled facilitator. Jossey-Bass Publishers, San Francisco

Soller A, Martnez-Mons A, Jermann P, Muehlenbrock M (2005) From mirroring to guiding: a review of state of the art technology for supporting collaborative learning. I. J Artif Intell Educ 15(4):261-290

Viller S (1991) The group facilitator: a cscw perspective. In: Bannon LJ, Robinson M, Schmidt K (eds) ECSCW. Kluwer, Dordrecht

Vivacqua A, Marques L, Ferreira M, de Souza J (2011) Computational indicators to assist meeting facilitation. Group Decis Negot 20:667-684

Wong Z, Aiken MW (2003) Automated facilitation of electronic meetings. Inf Manag 41(2):125-134 